\title{
Basic Compiler Algorithms for Parallel Programs *
}

\author{
Jaejin Lee and David A. Padua \\ Department of Computer Science \\ University of Illinois \\ Urbana, IL 61801 \\ $\{\mathrm{j}$-lee44, padua\}@cs.uiuc.edu
}

\author{
Samuel P. Midkiff \\ IBM T. J. Watson Research Center \\ P.O.Box 218 \\ Yorktown Heights, NY 10598 \\ smidkiff@us.ibm.com
}

\begin{abstract}
Traditional compiler techniques developed for sequential programs do not guarantee the correctness (sequential consistency) of compiler transformations when applied to parallel programs. This is because traditional compilers for sequential programs do not account for the updates to a shared variable by different threads. We present a concurrent static single assignment (CSSA) form for parallel programs containing cobegin/coend and parallel do constructs and post/wait synchronization primitives. Based on the CSSA form, we present copy propagation and dead code elimination techniques. Also, a global value numbering technique that detects equivalent variables in parallel programs is presented. By using global value numbering and the CSSA form, we extend classical common subexpression elimination, redundant load/store elimination, and loop invariant detection to parallel programs without violating sequential consistency. These optimization techniques are the most commonly used techniques for sequential programs. By extending these techniques to parallel programs, we can guarantee the correctness of the optimized program and maintain single processor performance in a multiprocessor environment.
\end{abstract}

*This work is supported in part by Army contract DABT6395-C-0097; Army contract N66001-97-C-8532; NSF contract ASC96-12099; and a Partnership Award from IBM. Jaejin Lee's work is partially supported by an IBM cooperative fellowship. This work is not necessarily representative of the positions or policies of the Army, Government or IBM Corp.

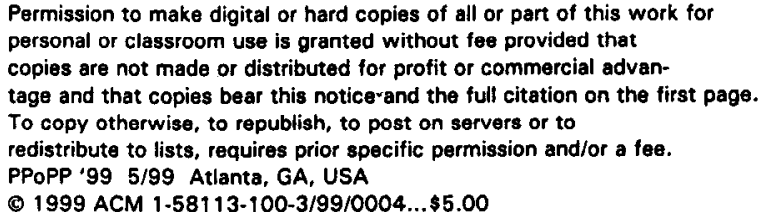

\section{Introduction}

Under the shared memory parallel programming model, all threads in a job can access a global address space. Communication between threads is via reads and writes of shared variables rather than explicit communication operations. Processors may access a shared variable concurrently without any fixed ordering of accesses, which leads to data races [5, 9] and non-deterministic behavior.

Data races and synchronization make it impossible to apply classical compiler optimization and analysis techniques directly to parallel programs because the classical methods do not account for updates to shared variables in threads other than the one being analyzed. Classical optimizations may change the meaning of programs when they are applied to shared memory parallel programs [23].

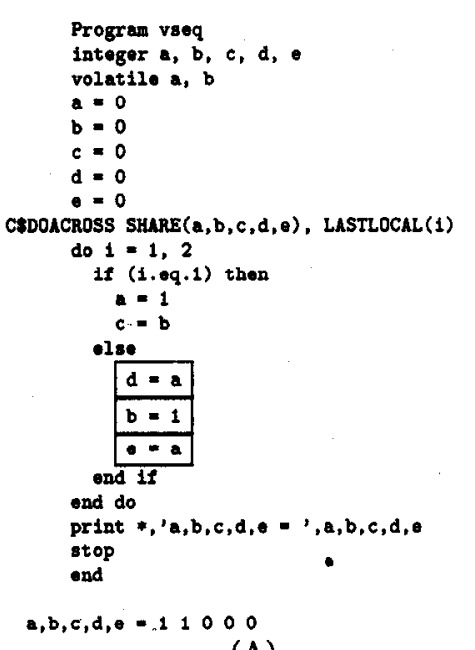

(A)

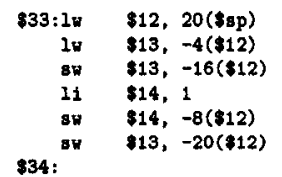

(B)

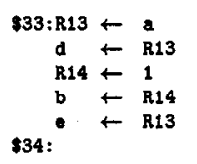

(C)
Figure 1. (A) A sample code. (B) An assembly code segment generated by the compiler for the boxed statements in (A) without any optimization switch on. (C) An intermediate code segment before register allocation that corresponds to the code in (B).

Consider the program in Figure 1(A) for an example of incorrectly applied classical optimization techniques. The 
MIPSpro Fortran 77 Compiler was used for compilation on an SGI challenge machine with 4 R4400 processors. During compilation, no optimization was enabled. The output ' $a, b, c, d, e=11000$ ' of the program on 2 processors in Figure $1(A)$ is not what the programmer would expect from an intuitive interpretation of the original program. By "the output the programmer expects," we mean the output attained by an execution that follows sequential consistency, which is the most intuitive and natural memory consistency model for programmers. The intuitive interpretation would lead us to assume that if $\theta$ is equal to 0 , then $c$ should be 1. The reason for this assumption is that if $e$ is equal to 0 , then $e=a$ should have executed before $a=1$, which means $b=1$ should have executed before $c=b$. We define sequential consistency [18] as follows.

\section{Definition 1.1 (Sequential Consistency)}

$A$ result of an execution of a parallel program $P$ is sequentially consistent if it is the same as the result of an execution where all operations were executed in some sequential order, and the operations of each individual thread occur in this sequence in the order specified by $P$.

Sequential consistency is what most programmers assume when they program shared memory multiprocessors, even if they do not know exactly what it is. An article supporting this argument at the hardware level is found in [12], where it is argued that future systems ought to implement sequential consistency as their hardware memory consistency model because the performance boost of relaxed memory consistency models does not compensate for the burden it places on system software programmers. We use sequential consistency as the correctness criterion for the execution of parallel shared memory programs.

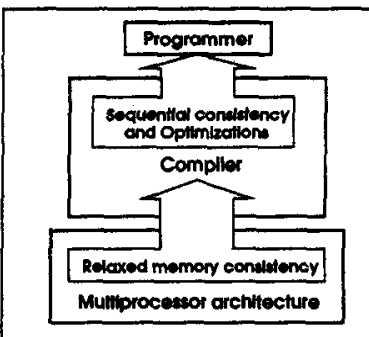

Figure 2. The compiler optimizations discussed in this paper together with fence generation algorithms present a sequentially consistent view to the programmer.

In this paper, we present analysis and optimization techniques which guarantee sequential consistency. We assume either that sequential consistency is enforced by the target machine or, if the target machine has a relaxed memory consistency model, that the back-end compiler inserts fences to enforce sequential consistency. One possible approach to enforce sequential consistency is to insert a fence instruction for each delay edge found by Shasha and Snir's delay set analysis [28]. A better method is discussed in [19] and is beyond the scope of this paper.

Our techniques make it possible to correctly handle the situations like the one in the example of Figure 1. Note that the problem arises from redundant load elimination (i.e., the reuse of symbolic register $R 13$ in the intermediate code in Figure 1(C) violates sequential consistency). The origin of the problem is that current commercial compilers were not implemented with sequential consistency or optimizations on sliared variables in mind.

Under the assumption of sequentially consistent execution of the original program, the defining characteristic of a correct compiler transformation in this paper is subset correctness.

\section{Definition 1.2 (Subset Correctness)}

A transformation is correct if the set of possible observable behaviors of a transformed program is a subset of possible observable behaviors of the original program.

An observable behavior of a program is a functional behavior of the program (i.e,, the program is treated as a black box and the programmer is interested in only its input and output). If a transformed program is sequentially consistent to the original program, it preserves subset correctness. But, not vice versa.

The remainder of the paper is organized as follows. In the next section, we describe Shasha and Snir's delay set analysis. In Section 3, we present an extension of the static single assignment (SSA) form that makes it possible to handle nested parallel do and cobegin/coend constructs and the post/wait synchronization. We describe algorithms for copy propagation and dead code elimination in Section 4 and global value numbering in Section 5. We present applications of the global value numbering technique, such as common subexpression elimination, redundant load/store elimination, and hoistable access detection (loop invariant detection) in Section 6. Section 7 presents related work, and Section 8 concludes our discussion.

\section{Delay Set Analysis}

Delays are the minimal ordering between shared variable accesses needed to guarantee sequential consistency. This minimal ordering is a subset of the ordering specified by the source program. It was shown by Shasha and Snir [28] that enforcing delays is sufficient to guarantee sequential consistency. Thus, instructions not related by the minimal ordering can be exchanged without affecting sequential consistency.

Consider a program control flow graph (CFG). Let $\mathbf{P}$ be the ordering specified by the source program on the set of variable accesses. $\mathbf{P}$ is the transitive closure of the ordering represented by the control flow edges in the CFG. Let $\mathbf{C}$ be a conflict relation on the variable accesses. Two memory accesses conflict if they are in different threads that could execute concurrently, reference the same memory location, and at least one is a write. Each element of $\mathbf{C}$ is represented as a bidirectional edge in the CFG. A critical cycle is a cycle of $\mathbf{P} \cup \mathbf{C}$ that has no chords in $\mathbf{P}^{\mathbf{1}}$. An edge $(u, v) \in \mathbf{P}$ is

\footnotetext{
${ }^{1}$ For two nonadjacent nodes $u$ and $v$ in the cycle, a chord is a
} 

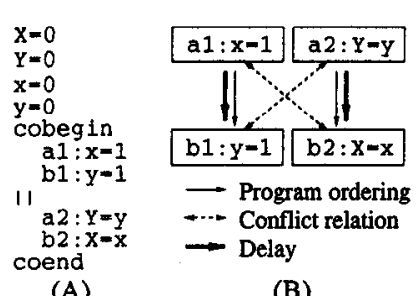

(B)

Figure 3. (A) A sample code. (B) The program edges (a1, b1) and (a2, b2) should be enforced to ensure sequentially consistent execution.

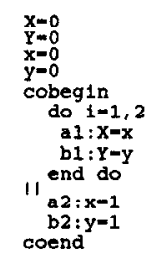

(A)

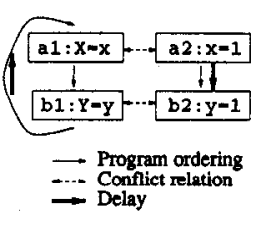

(B)
Figure 4. (A) A sample code gram edges (b1, a1) and (a2, b2) should be enforced to ensure sequentially consistent execution. with a loop. (B) The pro-

a critical pair if it occurs in a critical cycle. The critical pair relation is a minimal ordering relation that enforces sequential consistency in any execution of the program.

For example, the code shown in Figure $3(\mathrm{~A})^{2}$ gives a result $x=1, y=1, x=0$, and $Y=1$, which is not sequentially consistent, for the interleaving b1 a2 b2 a1. There is a critical cycle (b1, a2, b2, a1, b1) in this program and the $\mathbf{P}$ edges in the cycle are $(a 1, b 1)$ and $(a 2, b 2)$. Thus, the minimal ordering $\mathbf{D}=\{(\mathrm{a} 1, \mathrm{~b} 1),(\mathrm{a} 2, \mathrm{~b} 2)\})$ must be enforced as delays to guarantee sequential consistency.

One outcome of the code shown in Figure $4(A)$ is $x=1, y=1$, $X=0$, and $Y=1$. This is not sequentially consistent and is produced by the interleaving a1 a1 a2 b2 b1 b1. There is a critical cycle $(a 1, a 2, b 2, b 1, a 1)$ in this program and the $\mathbf{P}$ edges in the cycle are (b1, a1) and $(\mathrm{a} 2, \mathrm{~b} 2)$. Thus, the minimal ordering $\mathbf{D}=\{(\mathrm{b} 1, \mathrm{a} 1)$, (a2, b2) $\}$ ) must be enforced as delays. In other words, b1 in iteration $i$ must precede a1 in iteration $i+1$, and a2 and b2 cannot be reordered.

\section{Intermediate Representations}

We briefly discuss the concurrent control flow graph (CCFG) and the concurrent static single assignment (CSSA) form in this section. We also discuss how an early form of these representations $[20,21,22]$ has been extended to include both nested cobegin/coend and parallel do constructs with post/wait synchronization.

\subsection{Concurrent Control Flow Graph}

A basic block in a CCFG is a sequence of instructions where any branch is at the end and there is at most one shared variable access. If there is more than one shared variable access in an assignment, we decompose the assignment into several assignments so that each contains at most one shared variable access. The shared variable access is located at the beginning of a basic block for a read access and at the end for a write access. Two basic blocks conflict if their variable

$\mathbf{P}$ edge $(u, v)$.

${ }^{2}$ This example is taken from [28]. accesses conflict. Conflict edges join conflicting basic blocks and are bidirectional. Each direction of a conflict edge has a label. The label is $\delta^{t}$ if it is from a definition to a use, $\delta^{a}$ if it is from a use to a definition, and $\delta^{\circ}$ if it is from a definition to a definition. The labels appear at the head of conflict edges. A synchronization edge labeled $\sigma$ goes from post (S) to a wait (S) on the event variable $S$.

We have extended our early CCFG [20, 21, 22] to handle parallel do constructs. The definition of CCFG is in Ap pendix $A[20,21,22]$. Figure 6 shows the CCFG of the code in Figure 5. The region labeled $P$ represents a parallel do construct which is represented by a pdo node, followed by the representation of the loop body, followed by an endpdo node. Each parallel do construct is

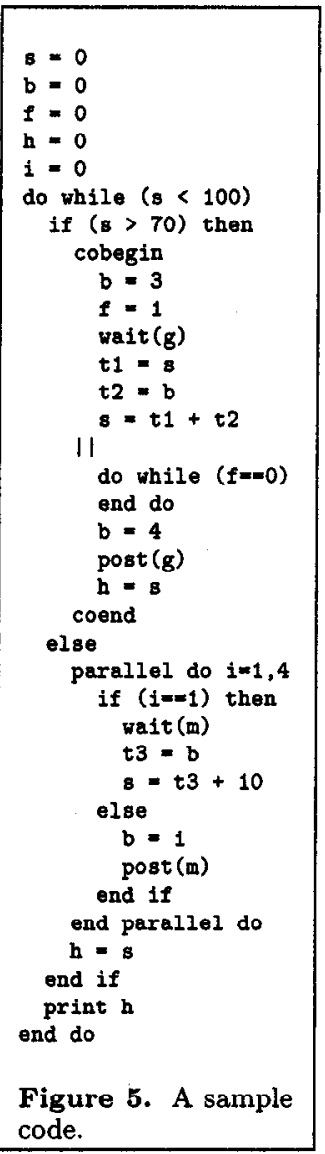
represented using two copies of the loop body. The two copies are treated as two different threads and summarize conflict and synchronization information between all the real threads created by the parallel do construct so that it is treated as a cobegin/coend construct. Loop private variables are renamed. $t 3$ in the original loop body [Figure 6] is renamed to $t 3^{\prime}$ in the copy of the loop body. The two ThreadEntry nodes in the parallel do construct contain assignments of two different constants, _c1 and _c2, to the loop index variable $i$ to indicate they are different threads. Also, the endpdo node contains an assignment for the final value of the loop index variable after execution of the parallel do construct. For the purposes of delay set analysis, we treat parallel loops as SPMD sections, and use the techniques ${ }^{3}$ of Krishnamurthy and Yellick $[15,16,17]$. Therefore, one copy of the loop body contains conflict edges to itself for each loop carried dependence. Each statement in a copy of the loop body corresponds to a dual statement in the other copy. When we apply a compiler optimization to a parallel do construct, it must be applied to both dual statements symmetrically. If this is impossible, we do not apply the optimization.

\footnotetext{
${ }^{3}$ They used two copies of a control flow graph of an SPMD program to perform delay set analysis.
} 


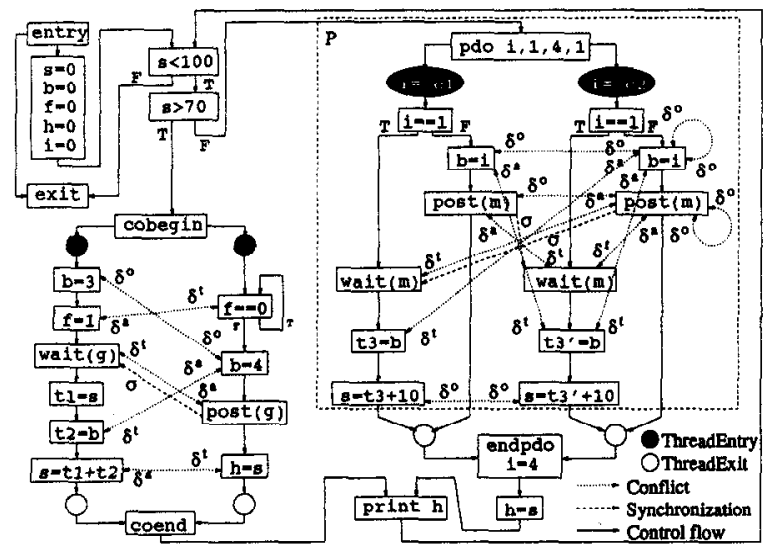

Figure 6. The concurrent control flow graph of the code in Figure 5.

\subsection{Concurrent Static Single Assign- ment Form}

The concurrent static single assignment (CSSA) form has these three confluence functions: $\phi$ (which is the same as the SSA $\phi$-function [7]) and two new functions: $\pi$ and $\psi$. These are defined as follows.

Definition 3.1 ( $\phi$-function)

$A \phi$-function of the form $\phi\left(v_{1}, v_{2}, \ldots, v_{n}\right)$ is placed at a node (except for a coend node or an endpdo node) where control flow edges join. $n$ is the number of incoming control flow edges at the node. The value of $\phi\left(v_{1}, v_{2}, \ldots, v_{n}\right)$ is one of the $v_{i}^{\prime} s$ and the selection depends on the control flow path followed by the program.

Definition 3.2 ( $\psi$-function)

A $\psi$-function for a shared variable $v$ has the form $\psi\left(v_{1}, v_{2}, \ldots, v_{n}\right)$, where $n$ is the number of threads merging at a coend node or an endpdo node where the $\psi$-function is placed. The value of $\psi\left(v_{1}, v_{2}, \ldots, v_{n}\right)$ is one of the $v_{i}^{\prime} s$ and the selection depends on the interleaving of statements in the threads merging at the node.

Definition 3.3 ( $\pi$-function)

$A \pi$-function of the form $\pi\left(v_{1}, v_{2}, \ldots, v_{n}\right)$ for a shared variable $v$ is placed where there is a use of the shared variable with $\delta^{t}$ conflict edges. $n$ is the number of reaching definitions to the use of $v$ through the incoming control flow edges and incoming confict $\delta^{t}$ edges. The value of $\pi\left(v_{1}, v_{2}, \ldots, v_{n}\right)$ is one of the $v_{i}^{\prime} s$. The selection depends on the interleaving of statements in the threads computing $v_{i}$ 's.

The definition of $\phi$-function in a CSSA form is the same as in the conventional SSA form. Cobegin/coend and parallel do constructs introduce new confluence points, e.g., coend and endpdo nodes. Instead of $\phi$-functions, $\psi$ functions, which are similar to those defined by Srinivasan et al. [31, 32], are placed at these new confluence points and summarize the interleaving of defining assignments of shared variables in cobegin/coend and parallel do constructs. However, $\phi$-assignments and $\psi$-assignments are insufficient to represent confluence of values because a definition of a shared variable may reach a use in a different thread. We place a $\pi$-function of a shared variable at the point where there is a use of the variable to summarize the confluence of values from different threads.

Like the SSA form for sequential programs, the CSSA form has the following properties:

- all uses of a variable are reached by exactly one (static) assignment to the variable

- for a variable, the definition dominates the uses if they are not arguments of $\phi$-, $\psi$-, or $\pi$-functions

Translating a CCFG of a parallel program into its CSSA form is a four-step process:

1. compute the execution ordering of basic blocks guaranteed by explicit synchronization

2. place $\phi$-assignments and $\psi$-assignments

3. rename variables

4. place $\pi$-assignments

To compute execution ordering, we start with a CCFG $G=(N, E, N$ type, Etype $)$. We compule a set $P_{r e c}[n]$ of nodes $m \in N$, which is guaranteed to precede $n \in N$ during execution. An iterative algorithm can be used to compute guaranteed execution ordering. The algorithm computes control and synchronization common ancestors of two nodes $[8,22]$. If there is a synchronization that depends on the loop index of a parallel do construct (c.g., synchronization using arrays) or that is control dependent on a node that uses the loop index of a parallel do, it is impossible to find statically the exact execution ordering with two copies of the loop body in the CCFG because we do not know which post will trigger which wait. We conservatively assume that there is no synchronization between two nodes that have such synchronization. However, there is the synchronization analysis technique developed by Callahan et al. [4] that can be applied to parallel do loops. It considers all the instances of statements in the iteration space and gives the ordering between them.

To insert $\phi$ - and $\psi$-assignments and rename variables, we can use the algorithm described by Cytron et al. [7]. At a coend and an endpdo nodes, $\psi$-assignments will be inserted instead of $\phi$-assignments.

The $\psi$-function can be removed (before renaming) if the arguments are ordered. The argument with the lowest precedence kills the definitions of all other arguments and can be used instead of the $\psi$-function. A similar optimization is used in [31]. 


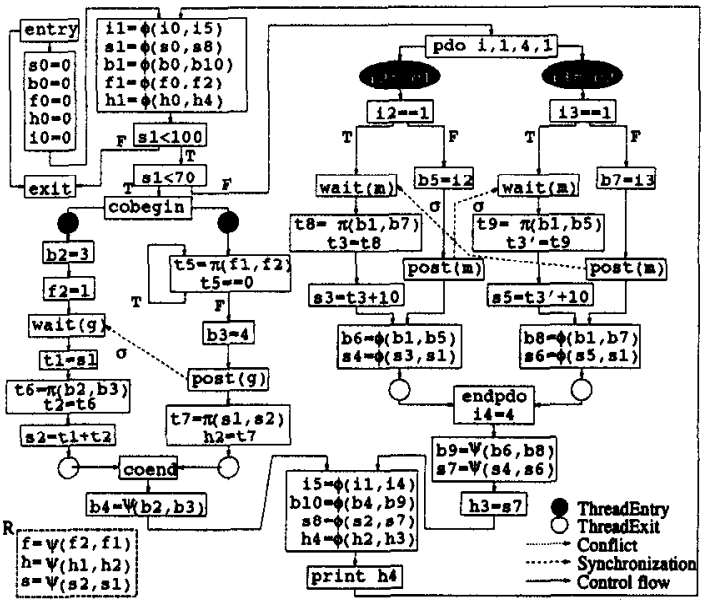

Figure 7. The CSSA form of the CCFG in Figure 6. For simplicity, conflict edges are omitted.

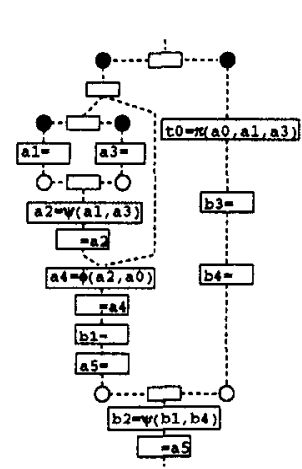

(A)

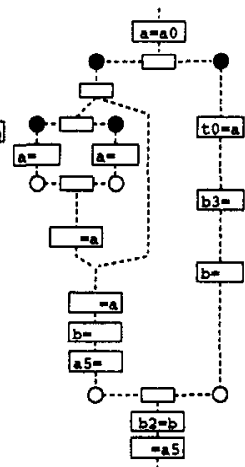

(B)
Figure 8. (A) A CSSA form. (B) After converting it to an executable intermediate form.

A reaching definition of a shared variable to its use may not be one of the arguments of the $\pi$-function for the use if it is killed by another reaching definition, or if its defining assignment executes after the use. We assign the $\pi$-function to a new temporary variable and insert this assignment immediately before the use. The use is replaced by this temporary variable. The algorithm for placing $\pi$-assignments is presented in Appendix B.

The CSSA form of the CCFG in Figure 6 is shown in Figure 7. For simplicity, conflict edges are omitted. The $\psi$ assignments in the lower left corner (in box $R$ ) were removed because they are redundant. Note that we do not place a $\psi$-assignment of the parallel do loop index $i$ in the endpdo node because it is killed by the assignment to $i$ in the endpdo node.

After optimizations and transformations on a CSSA form, we convert it into some executable form. Basically, we restore the original shared variable name and remove $\pi-, \psi_{-}$, and $\phi-$

\begin{tabular}{|c|c|c|c|}
\hline $\begin{array}{l}\text { In1tially } \\
k=0, t=0, k=0\end{array}$ & $\begin{array}{l}\text { In1tially } \\
a=0, t=0, k=0\end{array}$ & & : \\
\hline$: \quad:$ & : $\quad:$ & $\operatorname{ton} \pi\left(\mathbf{a} 0, \mathrm{a}_{1}\right) \vdots$ & $t 0=\pi(\mathrm{aO}, \mathrm{a1}):$ \\
\hline$t=a \quad:$ & $t=a$ & $t 1=t 0$ & $t 1=t 0$ \\
\hline $\begin{array}{c}: \quad a=1 \\
.\end{array}$ & $: a=1$ & $\vdots \quad$ a1=1 & $\vdots \quad$ a1=1 \\
\hline $\mathbf{k}=\mathbf{t} \quad:$ & $\mathbf{k}=\mathbf{a}:$ & k1=t1 $\vdots$ & $k 1=t 0$ \\
\hline : & : & $\vdots$ & : \\
\hline $\begin{array}{c}\text { Possible } \\
\text { Output } \\
(t=0, k=0) \\
(t=1, k=1)\end{array}$ & $\begin{array}{c}\text { Possibl. } \\
\text { Output } \\
(t=0, k=0) \\
(t=1, k=1) \\
(t=0, k=1)\end{array}$ & $\begin{array}{l}\text { Possible } \\
\text { Output } \\
(t 1=0, \quad k 1=0) \\
(t 1=1, \quad k 1=1)\end{array}$ & $\begin{array}{l}\text { Possible } \\
\text { Output } \\
(t 1=0, \quad k 1=0) \\
(t 1=1, k 1=1)\end{array}$ \\
\hline (A) & (B) & (C) & (D) \\
\hline
\end{tabular}

Figure 9. (A) A sample code before copy propagation. (B) The code in (A) after copy propagation. The result $t=0, k=1$ is not sequentially consistent. (C) The CSSA form of the code in (A). (D) The CSSA form in (C) after copy propagation. The result is sequentially consistent and does not violate subset correctness.

functions. An example is presented in Figure 8. Removing $\phi$-assignments for a local variable is the same as in the sequential SSA form. Because the $\psi$-assignment $\mathrm{b} 2=\psi(\mathrm{b} 1, \mathrm{~b} 4)$ is in the exit node of an outermost parallel construct, we replace the $\psi$-function with the original shared variable b and replace the definitions of its arguments b1 and b4 with b. The $\pi$-function in to $=\pi(20, a 1, a 3)$ is replaced with a, and then all definitions of its arguments a1 and a 3 are replaced with an assignment to a. Because the $\psi$-assignment $\mathrm{a}_{2}=\psi(\mathrm{a1}, \mathrm{a3})$ is not in the exit node of the outermost parallel construct and the definitions of its arguments are already replaced by a, it is just removed. Also, the use a2 that is not an argument of a $\psi$-, $\phi$ - or $\pi$-function is replaced by a. Since the definition a2 by $\psi$-assignment a2 $=\psi$ (a1, a3) reaches $a 4=\phi(a 2, a 0), a 4=\phi(a 2, a 0)$ is removed, and the use of $\mathrm{a} 4$ is replaced by $\mathrm{a}$. We insert an assignment $\mathrm{a}=\mathrm{a} 0$ at the beginning of the outermost parallel construct because the definition of argument a 0 comes from outside the outermost parallel construct. The untouched variables a0, a5, and b2 are treated as different variables from a.

\section{Copy Propagation and Dead Code Elimination}

For a copy assignment $t=a$, we can substitute a for $t$ at every use $u_{t}$ of $t$ if $[1,3]$ :

1. the assignment $t=\mathrm{a}$ is the only definition of $t$ reaching $u_{t}$

2. there are no assignments to a between $t=a$ and $u_{t}$ in any program execution

Classical copy propagation may violate sequential consistency in parallel programs. Consider the two threads in 
Figure $9(\mathrm{~A})$. After replacing $t$ with $a$ in $k=t$, we have the code in Figure $9(\mathrm{~B})$ which can produce an incorrect result $t=0, k=1$. This copy propagation violates sequential consistency. The other thread modifies the variable a between $t=a$ and $\mathrm{k}=\mathrm{t}$.

In the CSSA form [Figure 9(C)], if the propagated variable is shared, a local copy is always used for copy propagation because of $\pi$-assignments. Thus, the case in Figure 9(B) never occurs [Figure 9(D)]. Also, the case where the substituted variable is shared never occurs if we do not allow copy propagation when the substituted variable is in a $\phi$, $\psi$-, or $\pi$-function. Thus, the conventional copy propagation can be directly used if we do not propagate to a $\phi-, \psi$-, or $\pi$-functions and the parallel program is in CSSA form [Figure $9(\mathrm{C})$ and (D)].

An instruction $I$ is dead if the values it computes are not used in any executable path starting at $I$ [25]. Some optimizations, such as copy propagation, produce dead instructions. In the sequential case, we mark essential instructions and follow use-def chains for the variables used in the instructions. If an instruction is in the chain, it cannot be dead coded. The essential instructions are those that might affect observable behaviors of the program, such as input/output, function call, and control flow instructions ${ }^{4}[3,25]$. Dead code elimination for parallel programs is essentially the same as the sequential case but we must consider interactions between threads through shared variables. The CSSA form is useful for dead code elimination in parallel programs because it contains inter-thread use-def chains. The dead code elimination algorithm for parallel programs in CSSA form is the same as the sequential one except definitions from another thread are considered.

\section{Global Value Numbering}

The equivalence of variables in sequential programs is defined by Alpern et al. in [2]. This definition is used as a basis for their SSA based value partitioning algorithm. We use the same definition for equivalence of variables in parallel programs.

\section{Definition 5.1 (Equivalence of Variables)}

Two variables are said to be equivalent at a point $p$ if they contain the same values whenever control reaches $p$ during any possible execution of the program.

Because the notion of equivalence contains dynamic instances of variables, Alpern et al. defined a congruence relation of nodes in a value graph to detect equivalence statically [2]. There is a node in the value graph for each binary operation in the program. When the result of the operation is assigned to a variable, the variable name is used to label the node. Each edge is labeled by the position of the

\footnotetext{
${ }^{4}$ We cannot dead code infinite loop because it affects observable behavior of the program.
}

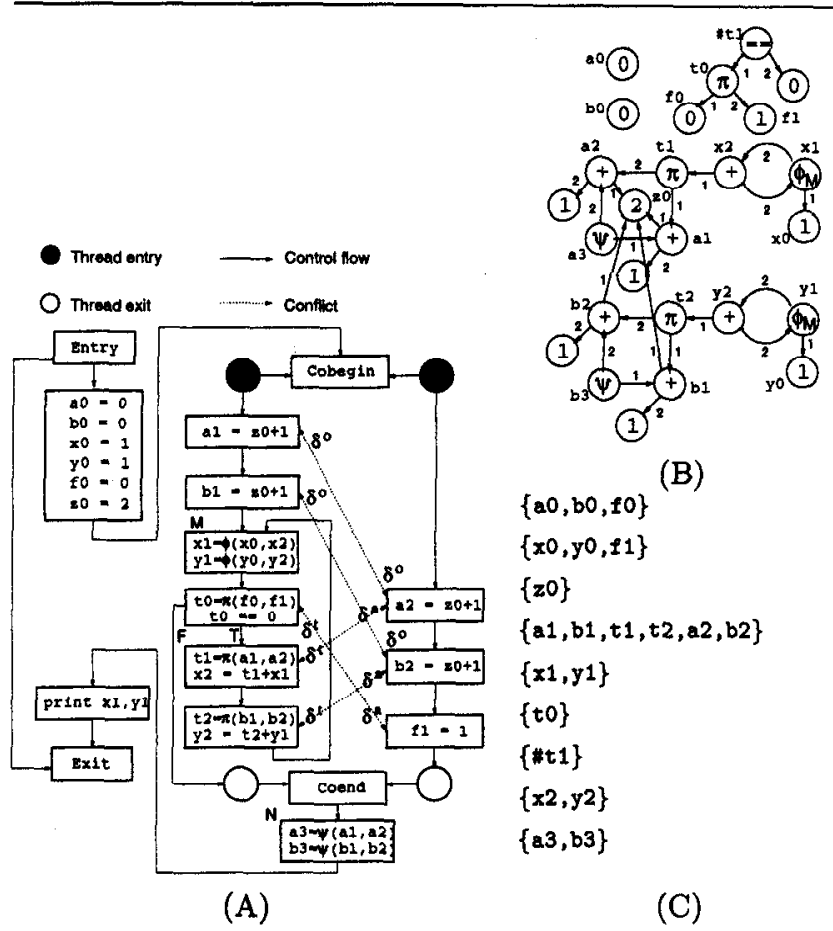

Figure 10. (A) A CSSA form. (B) The value graph. (C) After partitioning. The variables that have the same value numbers are in the same partition. \#t 1 is a value number for the expression to $=0$ that does not define any variable.

corresponding operand with respect to the operation. The operation label $\phi$ of a node that corresponds to a $\phi$-function is subscripted by the CCFG node where the $\phi$-function is placed. An example of the value graph of a CSSA form is in Figure 10.

Following Alpern et al., we define congruence on the value graph of a parallel program as follows:

\section{Definition 5.2 (Congruence)}

- two nodes are congruent if their operation labels are the same and are not $\pi$ or $\psi$, and their departing edges with the same labels lead to congruent nodes.

- a node with the operation label $\pi$ or $\psi$ is congruent to its arguments if all arguments are congruent to each other.

The first condition is the same as that of \begin{tabular}{c}
\hline$\vdots$ \\
$t 0=\pi(a 0, a 1)$ \\
$\vdots$ \\
$t 1=\pi(b 0, b 1)$ \\
$\vdots$ \\
Figure 11.
\end{tabular} the sequential case. To explain the reason behind the second condition, consider Figure 11. Assume congruence between a 0 and bo and between $a 1$ and b1. Also, assume the value of to is a1 in execution. Because the value of a $\pi$-function can be any of of its arguments, the value of $t 1$ will not necessarily be that of $\mathrm{b} 1$ in the execution. Thus, the first condition cannot be the condition that entails congruence between to and $t 1$. We assume a $\pi$ - 
function or a $\psi$-function is congruent to its arguments when all the arguments are congruent. Intuitively, a $\pi$-function has the same value as its arguments if all the arguments have the same value.

Because of the subscripts, two $\phi$-functions in different CCFG nodes are never considered congruent. If the corresponding condition expression for a $\phi$-function is known, the condition can be incorporated to determine congruence of $\phi$-functions in different CCFG nodes [2].

The concurrent global value numbering (CGVN) algorithm is an optimistic partitioning algorithm to find congruent variables. The algorithm is an extension of a global value numbering algorithm for sequential programs that was developed by Cooper and Simpson $[6,29]$. The global value numbering is essentially the same as Alpern et al.'s value graph partitioning [2].

The concurrent global value numbering algorithm is shown in Figure 12. In concurrent global value numbering, all variables (nodes in the value graph) are initially placed in the same partition and therefore assumed to be congruent. This assumption is refined until a fixed point is reached. The algorithm proceeds in a sequence of steps. In each step, all the nodes in the CCFG are traversed in reverse post order of a spanning tree. The process continues until a fixed point is reached. In step $i$, (i) a variable (node), whose operation label is not $\pi$ or $\psi$, is in the same partition as another node if they were in the same partition in step $\mathrm{i}-1$, and the corresponding destination of the edges leaving the nodes were pairwise in the same partition in step i-1, or (ii) if the operation label is $\pi$ or $\psi$, and its arguments were all in the same partition in step $\mathrm{i}-1$, the node is still in the same partition as its arguments.

Without loss of generality, we assume that all definitions in a CSSA form are in one of the following forms: $\quad \mathrm{x}=\mathrm{y}, \mathrm{x}=\mathrm{y}$ op $\mathrm{z}, \mathrm{x}=\phi\left(v_{1}, \ldots, v_{m}\right), \mathrm{x}=\psi\left(v_{1}, \ldots, v_{m}\right)$, and $\mathrm{x}=\pi\left(v_{1}, \ldots, v_{m}\right)$, where $\mathrm{x}$ is a variable and $\mathrm{y}$ and $\mathrm{z}$ are either constants or variables. In this algorithm, a value number is a variable name. The function $\operatorname{LookUp}\left(\right.$ index $\left._{e}, v\right)$ searches a hash table with a variable $v$ and the hash index index $x_{e}$ of its defining expression $e$. If the value number of the hash index is not defined (i.e., T), it assigns the value number $v$ to index $x_{e}$ and returns $v$. Otherwise, it returns the value number of index. The array $V$ alNum maps a variable or a constant to its value number. In the first for loop, the algorithm gives the same value number to all the nodes of the value graph. The partitions of variables in Figure 10(B) after the global value numbering is in Figure $10(\mathrm{C})$.

Similar to the sequential case [2], we can show that the following theorem and corollary hold for the CSSA form by using the notion of active variables.

Definition 5.3 (Active variable)

A variable is active at a moment during execution if and

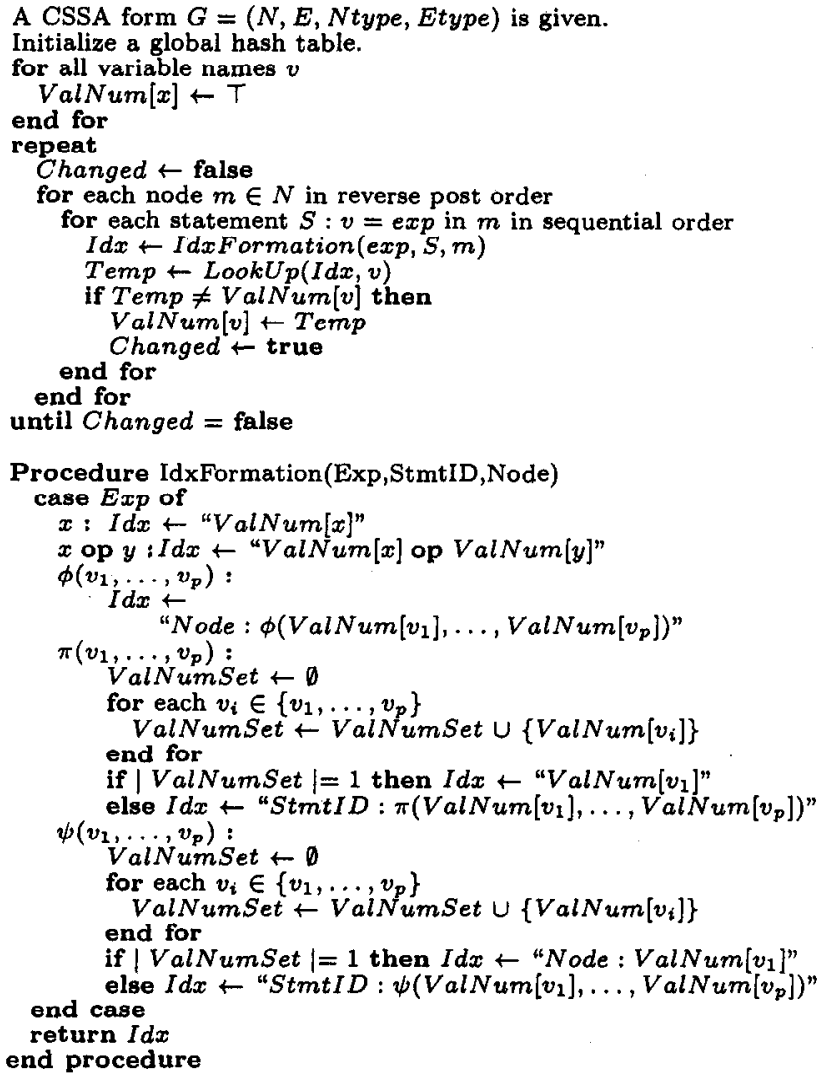

Figure 12. Concurrent Global Value Numbering Algorithm. A variable in between a pair of double quotes stands for the corresponding string converted from the value of the variable.

only if it satisfies one of the following two conditions:

- the defining assignment of the variable is not contained in an innermost loop that is currently executing, and has already been executed.

- the innermost loop containing the defining assignment of the variable is currently executing and the assignment has already been executed during the current iteration.

Theorem 5.4 (Equivalence)

Two active and congruent variables have the same value in any possible execution of a program in CSSA form.

Proof: See Appendix C.

\section{Corollary 5.5}

Two congruent variables are equivalent at a point $p$ if their defining assignments dominate $p$.

Proof : If the assignment to a variable dominates a point, then the variable must be active at the point. By Theorem 5.4, they are equivalent.

We will use the result of Corollary 5.5 for our extension of common subexpression elimination in the next section. 
CGVN treats $\pi$-functions and $\psi$-functions conservatively in the presence of imprecisely introduced arguments due to imprecise synchronization analysis. Because two $\pi$-functions ( $\psi$-functions) are congruent if and only if all their arguments are congruent and because the value of a $\pi$-function ( $\psi$-function) will be the value of one of its arguments, an imprecise argument cannot affect the value of those two $\pi$ functions ( $\psi$-functions).

\section{Applications of Concurrent Global Value Numbering}

In this section, we discuss some applications of the concurrent global value numbering.

\subsection{Common Subexpression Elimina- tion}

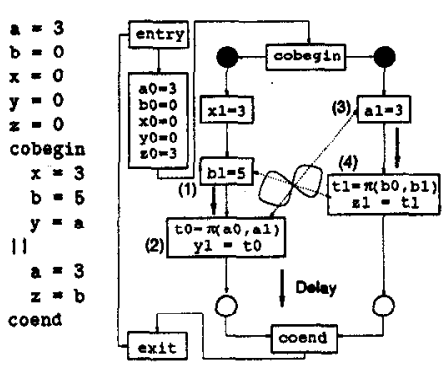

(A)

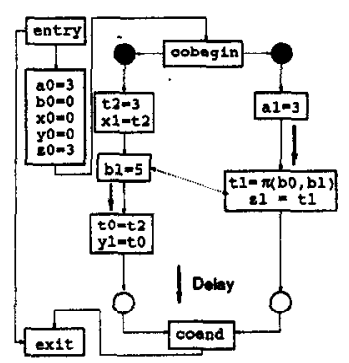

(C)

Figure 13. Since there exists a critical cycle $((1),(2),(3),(4),(1))$ (i.e., the 8-like shape in (B)), we have to enforce delays on program edges ((1),(2)) and ((3),(4)) to get sequentially consistent execution. After CSE is done in (C), the CSE violates sequential consistency. The result $y 1=3, b 1=5, a 1=3$, and $z 1=0$ of the code in (C) is not possible from the code in (B).

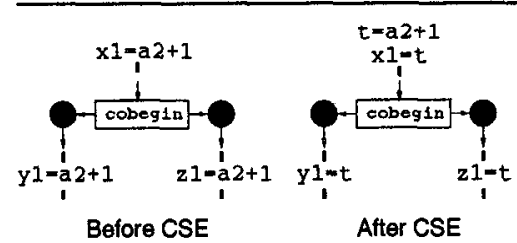

Figure 14. Because $x 1=a 2+1$ dominates $y 1=a 2+1$ and $z 1=a 2+1$, we can substitute $a 2+1$ with $t$ in $\times 1=a 2+1$, $y 1=a 2+1$ and $z 1=a 2+1$ after inserting an assignment $t=a 2+1$ immediately before $x 1=a 2+1$. CSE introduced two new shared variable accesses (i.e., $t$ ).

Common subexpression elimination (CSE) is one of the most common optimization techniques for sequential programs [25]. However, it cannot be applied directly to parallel programs. Consider the code in Figure 13(A) and its CSSA form in (B). Because a 0 and a1 are congruent, to is congruent to $a 0$ and a1. Also, $x 1$ is congruent to $a 0$ and $a 1$. Thus, $x 1$ and to are congruent. Because $x 1=3$ dominates t $0=\pi(a 0, a 1), x 1$ and to have the same value at the point immediately after $t 0=\pi(a 0, a 1)$. Thus, if we apply classical CSE to $x 1$ and $t 0$, we obtain the program in Figure 13(C). Doing CSE in this case is equivalent to moving the statement $t 0=\pi\left(a_{1}, a_{2}\right)$ to the point immediately after $x 1=3$ and executing it together with $\times 1=3$. This makes the result of the program the same as if the order of execution were $t 0=\pi(a 0, a 1), y 1=t 0, a 1=3$, $t 1=\pi(b 0, b 1), z 1=t 1, b 1=5$. The result from this execution does not correspond to any result from the original program, and therefore applying classical CSE leads to incorrect results. The problem can be identified by applying delay set analysis, which would show that $t 0=\pi(a 0, a 1)$ must execute after $b 1=5$, and $a 1=3$ must execute before $t 1=\pi(b 0, b 1)$. That is, the delay edges in Figure 13(B) must be enforced. This order is violated by the execution sequence associated with the CSE translation.

As seen in Figure 14, applying CSE may introduce a shared variable access ( $t$ in Figure 14). This could be unprofitable depending on the size of the common subexpression, because shared variable accesses take more time than local variable accesses.

Thus, in order to do CSE in parallel programs, we must make use of delay set analysis and we must consider profitability. For two congruent variables $v$ and $w$, we apply CSE when the following conditions hold ( $S_{v}$ and $S_{w}$ denote the defining assignments of $v$ and $w$ in the CSSA form):

- (equivalence condition) $S_{v}$ dominates $S_{w}$

- (sequential consistency condition) there does not exist a statement $X \neq S_{v}$ such that there is a delay edge $\left(S_{w}, X\right)$ or $\left(X, S_{w}\right)$

- (profitability condition) application of CSE is profitable

In some cases, doing CSE does not reduce or increase the execution time of a program. As seen in Figure 15, doing CSE in Thread 1 will not reduce or increase the execution time of the program. However, doing CSE in the critical thread Thread 2 will be beneficial.

After CGVN is done on the CSSA form, CSE is applied to the CSSA form. The CSE algorithm is called concurrent common subexpression elimination (CCSE) [Figure 16].

\subsection{Redundant Load/Store Elimination}

Register allocation is an important optimization for sequential programs because the number of machine registers are limited and because register operations are much faster than memory operations. The conventional register allocation algorithm would work well on parallel programs were it not for optimizations, such as loop invariant code motion and redundant load elimination (RLE) applied in the middle of or before the register allocation phase. The sequential consistency violation example in Figure 1 comes from RLE. The redundant store elimination (RSE) removes store operations 
Let $G=(N, E, N$ type, Etype) be the given CCFG in CSSA form. Done is an array that indicates if a variable is already processed or not. Temp is an array that contains a temporary variable used in $\mathrm{CSE}$ for each variable if there is one for the variable. InnermostPC is an array that contains the innermost parallel construct for each node in the CSSA form. First $(\mathcal{W})$ returns the first element in the list $\mathcal{W}$ and remove it from $\mathcal{W}$. Children[n] is the set of children of $n$ in the dominator tree. The algorithm traverses the dominator tree in breadth first manner.

Do the delay set analysis on the CSSA form.

Do the concurrent global value numbering on the CSSA form.

Construct the dominator tree of the CSSA form.

let $r$ be the root of the dominator tree.

$W+r$

for each node $n \in N$ in the CSSA form

let $h$ be the innermost parallel construct that contains $n$

Innermost $P C[n] \leftarrow h$

end for

for each variable $v$ in the CSSA form

Done $[v] \leftarrow$ false

Temp $[v] \leftarrow T$

end for

while $W \neq 0$

$n \leftarrow \operatorname{First}(\mathcal{W})$

$\mathcal{W} \leftarrow \mathcal{W} \cup$ Children $[n]$

for each variable $v$ defined in $n$ in sequential order

if Done $[v]=$ false then

Done $[v] \leftarrow$ true

let $P_{v}$ be the partition where $v$ belong

for each $r \in P_{v} \wedge$ Done $[r]=$ false

let $S_{v}$ be the defining statement $v=e$ of $v$

let $S_{r}$ be the defining statement $r=e_{r}$ of $r$

if $e_{r}$ is a $\phi$ - or $\psi$-function then

Done $[r] \leftarrow$ true

else

if Innermost $P C[n]=$ Innermost $P C\left[n_{r}\right]$ then

let $n_{r}$ be the defining node of $r$

if $n$ dominates $n_{r}$ then

if there does not exist a statement $X$ such that

there is a delay $\left(S_{r}, X\right)$ or $\left(X, S_{r}\right)$ then

if $T e m p[v]=T$ then

Generate a new local temporary variable $t$ and place an assignment $t=e$

immediately before $S_{v}$. Substitute $t$ for $e$ in $S_{v}$ $T e m p[v] \leftarrow t$

Substitute $T e m p[v]$ for $e_{r}$ in $S_{r}$

end for

Done $[r] \leftarrow$ true$$
\text { end for }
$$

end while

Figure 16. Concurrent Common Subexpression Elimination Algorithm.

that write the same value that was previously written to the same memory location, such that memory traffic is reduced. If we treat the variable in the load or store instruction as an expression, the CCSE can be used for both RLE and RSE of parallel programs without violating any correctness criteria. The CSSA form of the example in Figure 1 is shown in Figure 17. If we apply CGVN in this form, $t 1$ and $t 2$ are not congruent, nor are $t 4$ and $t 5$. The reason is that $a 0$ and $a 3$ are not congruent, nor are a 0 and a1. Thus, the application of RLE does not change the program.

\subsection{Hoistable Access Detection}

Loop invariant code motion is another common optimization technique for sequential programs. Consider the code in Figure 18. The statement $x=a+1$ in the while loop is a loop invariant in the classical sense [Figure 18(A)]. However, if we move the statement outside the loop [Figure 18(B)], it

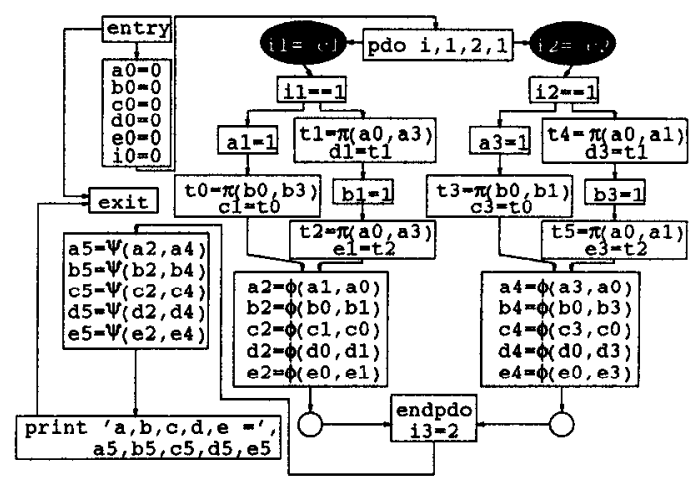

Figure 17. The CSSA form of the example in Figure 1. If we apply concurrent global value numbering in this form, $t 1$ and $t 2$ are not congruent and nor are $t 4$ and $t 5$. Thus, we cannot apply redundant load application in this case. For simplicity, conflict edges are omitted.

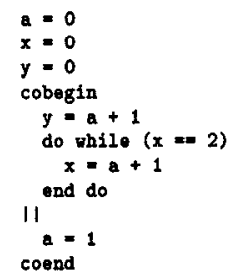

(A)
(B)

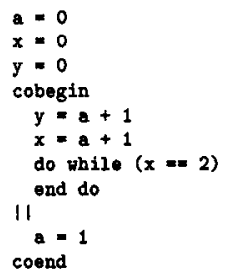

Figure 18. (A) Before loop invariant code motion. (B) After loop invariant code motion. The wh1le loop was made infinite.

might make the loop infinite. This does not happen in the original program (i.e., the loop invariant code motion violates subset correctness). For conventional compilers doing single threaded analysis, it is common to move a load instruction of a variable in a loop outside the loop if the load instruction is loop invariant. However, if we do not consider modification of the variable by another thread, moving load instructions is another source of sequential consistency violations. We define a hoistable access for parallel programs that is a counterpart to a loop invariant in sequential programs.

\section{Definition 6.1 (Hoistable access)}

We call any computation in a loop that can be moved outside the loop without affecting the meaning of the given program a hoistable access.

Similar to CSE, the notion of hoistable accesses also involves code motion. Thus, we must guard against sequential consistency violations. We mark a $\pi$-assignment in a loop hoistable if moving it outside the loop does not violate ordering enforced by delays and if all its arguments are congruent. If all the variable accesses in an instruction in a loop are hoistable, then the instruction can be moved outside the loop. The 


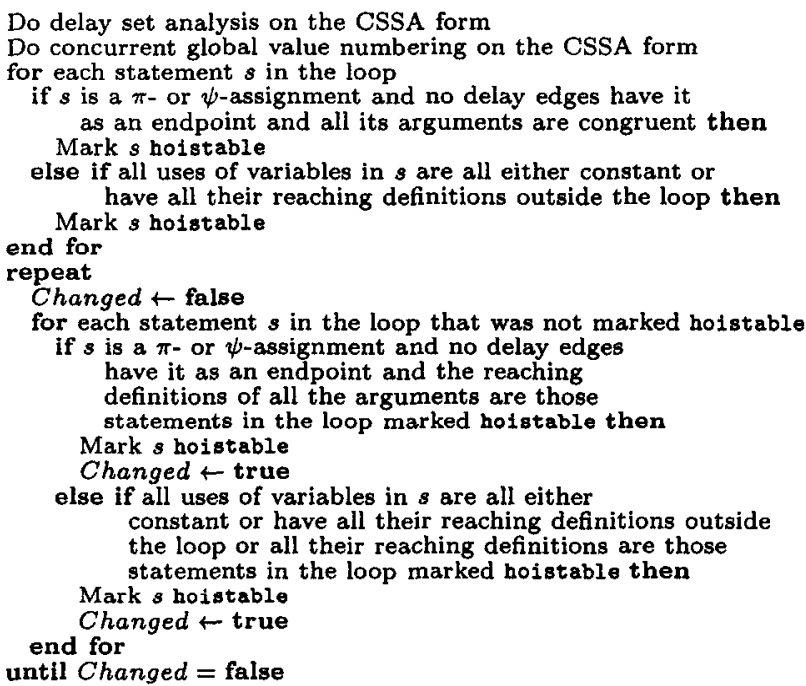

Figure 19. Hoistable Access Detection Algorithm.

hoistable access detection algorithm based on CSSA form is in Figure 19.

\section{Related Work}

Despite the increasing importance of compiling parallel programs, there has been little work in the field. A static single assignment (SSA) form called parallel static single assignment form (PSSA), for parallel programs was proposed by Srinivasan et al. $[30,34,31,32]$. PSSA was developed for the PCF Parallel Fortran [11] parallel sections construct with copy-in/copy-out semantics. With copy-in/copy-out semanlics, each thread receives its own copy of the shared variables at a fork point and can modify only its own local copy. Thus, the result of a parallel execution does not depend on a particular interleaving of statements in the parallel program and the PSS $\Lambda$ form cannot handle parallel programs with truly shared memory semantics.

Sarkar and Simons proposed a parallel program graph (PPG) [27] that subsumes program dependence graphs (PDGs) [10] and conventional control flow graphs (CFGs) [1]. Sarkar [26] developed reaching definitions analysis on PPGs for deterministic parallel programs.

Krishnamurthy and Yelick developed $O\left(n^{3}\right)$ time delay set analysis algorithm for SPMD programs. They used two copies of a control flow graph of a parallel SPMD program and inserted conflict edges in between these two copies. The graph is used for detecting critical cycles [28]. Also, they present optimization techniques such as communication optimization, message pipelining, and eliminating remote accesses through data reuse for SPMD programs $[15,16,17]$.

Midkiff, Padua, and Cytron [24] extended Shasha and Snir's delay set analysis [28] to subscripted variables (i.e., arrays) by using a statement instance level flow graph.

Knoop, Steffen, and Vollmer [14, 13] presented a unidirectional bit vector data flow analysis frame work based on abstract interpretation and showed that it can be used for code motion and partial dead code elimination. Their framework handles the cobegin/coend construct but not synchronization mechanisms.

Lee, Midkiff, and Padua proposed the CSSA form based on CCFGs for parallel programs with cobegin/coend construct and the post/wait synchronization mechanism. They have extended Wegman and Zadeck's [33] sparse conditional constant propagation algorithm for parallel programs $[20,21,22]$. The CCFG and CSSA form has been extended to handle the parallel do construct in this paper.

Previous work in this field has concentrated mostly on techniques for analyzing parallel programs. There is little work on optimization techniques. We have presented several optimization techniques for parallel programs that extend common optimization techniques for sequential programs such as common subexpression elimination, copy propagation, dead code elimination, loop invariant code motion, and redundant load/store elimination. Most of the previous work either ignores or avoids using sequential consistency as a correctness criterion. Our analysis and optimization techniques guarantee sequential consistency. In addition, our work is more general than previous work in representing parallel constructs such as cobegin/coend, parallel do, and post/wait synchronization constructs and in dealing with non-deterministic parallel programs. The CSSA form handles parallel programs with truly shared memory semantics that cannot be handled with copy-in/copy-out semantics.

\section{Conclusions}

We proposed two intermediate representations, the concurrent control flow graph and the concurrent static single assignment form, for shared memory parallel programs with nested cobegin/coend and parallel do constructs and post/wait synchronization mechanisms. Like the conventional SSA form, the CSSA form has the following properties:

- all uses of a variable are reached by exactly one (static) assignment to the variable.

- for a variable, the definition dominates the uses if they are not arguments of $\phi$-, $\psi$-, or $\pi$-functions

Sequential consistency and subset correctness are our correctness criteria for execution and compiler transformations. We have presented a conservative analysis technique called concurrent global value numbering that is used to detect equivalent variables in parallel shared memory programs. We have extended some classical scalar optimization techniques to guarantee sequential consistency on parallel pro- 
grams in CSSA form. These optimization techniques include:

- copy propagation

- dead code elimination

- concurrent common subexpression elimination

- redundant load/store elimination

- hoistable access detection

We believe that the analysis and optimization techniques and the intermediate representations presented in this paper are the first step towards an optimizing compiler for parallel programs.

\section{References}

[1] Alfred V. Aho, Ravi Sethi, and Jeffrey D. Ullman. Compilers: Principles, Techniques, and Tools. Addison Wesley, 1986.

[2] Bowen Alpern, Mark N. Wegman, and F. Kenneth Zadeck. Detecting equality of variables in programs. In Conference Record of the Fifteenth ACM Symposium on Principles of Programming Languages, pages 1-11, January 1988.

[3] Andrew W. Appel. Modern Compiler Implementation in Java. Cambridge University Press, New York, 1998.

[4] David Callahan, Ken Kennedy, and Jaspal Subhlok. Analysis of event synchronization in a parallel programming tool. In Proceedings of the 2nd ACM SIGPLAN Symposium on Principles and Practice of Parallel Programming (PPOPP), pages 21-30, March 1990.

[5] Jong-Deok Choi and Sang Lyul Min. Race frontier: Reproducing data races in parallel-program debugging. In Proceedings of the 3rd ACM SIGPLAN Symposium on Principles and Practice of Parallel Programming (PPOPP), pages 145-154, April 1991.

[6] Keith Cooper and Taylor Simpson. SCC-based value numbering. Technical Report CRPC-TR95636-S, Center for Research on Parallel Computation, Rice University, October 1995.

[7] Ron Cytron, Jeanne Ferrante, Barry K. Rosen, and Mark N. Wegman. Efficiently computing static single assignment form and the control dependence graph. ACM Transactions on Programming Languages and Systems, 13(4):451-490, October 1991.

[8] Perry A. Emrath, Sanjoy Ghosh, and David A. Padua. Event synchronization analysis for debugging parallel programs. In Proceedings of Supercomputing '89, pages 580-588, 1989.

[9] Perry A. Emrath, Sanjoy Ghosh, and David A. Padua. Detecting nondeterminacy in parallel programs. IEEE Software, pages 69-77, January 1992.

[10] Jeanne Ferrante, Karl J. Ottenstein, and Joe D. Warren. The program dependence graph and its use in optimization. ACM Transactions on Programming Languages and Systems, 9(3):319-349, July 1987.

[11] The Parallel Computing Forum. PCF parallel fortran extensions. Fortran Forum, 10(3), September 1991.

[12] Mark D. Hill. Multiprocessors should support simple memory-consistency models. IEEE Computer, pages 28-34, August 1998.
[13] Jens Knoop. Eliminating partially dead code in explicitly parallel programs. Theoretical Computer Science, 196(1-2):365-393, April 1998.

[14] Jens Knoop, Bernhard Steffen, and Jürgen Vollmcr. Parallelism for free: Efficient and optimal bitvector analysis for parallel programs. ACM Transactions on Programming Languages and Systems, 18(3):268-299, May 1996.

[15] Arvind Krishnamurthy and Katherine Yelick. Optimizing parallel SPMD programs. In Seventh Annual Workshop on Languages and Compilers for Parallel Computing, August 1994.

[16] Arvind Krishnamurthy and Katherine Yelick. Optimizing parallel programs with explicit synchronization. In Proceedings of the ACM SIGPLAN 1995 Conference on Programming Language Design and Implementation (PLDI), pages 196-204, June 1995.

[17] Arvind Krishnamurthy and Katherine Yelick. Analyses and optimizations for shared address space programs. Journal of Parallel and Distributed Computing, 38:139$144,1996$.

[18] Leslie Lamport. How to make a multiprocessor computer that correctly executes multiprocess programs. IEEE Transactions on Computers, C-28(9):690-691, September 1979.

[19] Jaejin Lee. Compilation Techniques for Parallel Shared Memory Programs. PhD thesis, University of Illinois at Urbana-Champaign, 1999. In preparation.

[20] Jaejin Lee, Samuel P. Midkiff, and David A. Padua. Concurrent static single assignment form and concurrent sparse conditional constant propagation for explicitly parallel programs. Technical Report TR\#1525, CSRD, University of Illinois at Urbana-Champaign, July 1997.

[21] Jaejin Lee, Samuel P. Midkiff, and David A. Padua. Concurrent static single assignment form and constant propagation for explicitly parallel programs. In Proceedings of The 10th International Workshop on Languages and Compilers for Parallel Computing, number 1366 in Lecture Notes in Computer Science, pages 114-130. Springer, August 1997.

[22] Jaejin Lee, Samuel P. Midkiff, and David A. Padua. A constant propagation algorithm for explicitly parallel programs. International Journal of Parallel Programming, 26(5):563-589, 1998.

[23] Samuel. P. Midkiff and David. A. Padua. Issues in the optimization of parallel programs. In Proceedings of the 1990 International Conference on Parallel Processing (ICPP), Vol. II Software, pages 105-113, August 1990.

[24] Samuel P. Midkiff, David A. Padua, and Ron Cytron. Compiling programs with user parallelism. In Languages and Compilers for Parallel Computing, pages 402-422, 1990.

[25] Steven S. Muchnick. Advanced Compiler Design and Implementation. Morgan Kaufmann, 1997.

[26] Vivek Sarkar. Analysis and optimization of explicitly parallel programs using the parallel program graph representation. In The Tenth Annual Workshop on Languages and Compilers for Parallel Computing, number 1366 in Lecture Notes in Computer Science, pages 94113. Springer, August 1997. 
[27] Vivek Sarkar and Barbara Simons. Parallel program graphs and their classification. In The Sixth Annual Workshop on Languages and Compilers for Parallel Computing, volume 768 of Lecture Notes in Computer Science. Springer-Verlag, August 1993.

[28] Dennis Shasha and Marc Snir. Efficient and correct execution of parallel programs that share memory. $A C M$ Transactions on Programming Languages and Systems, 10(2):282-312, April 1988.

[29] Loren Taylor Simpson. Value-Driven Redundancy Elimination. PhD thesis, Department of Electrical and Computer Engineering, Rice University, April 1996.

[30] Harini Srinivasan. Optimizing explicitly parallel programs. Master's thesis, Department of Computer Science and Engineering, Oregon Graduate Institute of Science and Technology, July 1994.

[31] Harini Srinivasan and Dirk Grunwald. An efficient construction of parallel static single assignment form for structured parallel programs. Technical Report CU-CS564-91, University of Colorado at Boulder, December 1991.

[32] Harini Srinivasan, James Hook, and Michael Wolfe. Static single assignment for explicitly parallel programs. In Proceedings of the 20th ACM Symposium on Principles of Programming Languages (POPL), pages 260272, January 1993.

[33] Mark N. Wegman and F. Kenneth Zadeck. Constant propagation with conditional branches. $A C M$ Transactions on Programming Languages and Systems, 13(2):181-210, April 1991.

[34] Michael Wolfe and Harini Srinivasan. Data structures for optimizing programs with explicit parallelism. In Proceeedings of the First International Conference of the Austrian Center for Parallel Computation, September 1991.

\section{Appendix A}

Concurrent Control Flow Graph

A concurrent control flow graph (CCFG) is a directed graph $G=$ ( $N, E, N$ type, Etype) such that,

口 $N$ is the set of nodes in $G$. Each node is a basic block.

$\square E=E_{\text {cont }} \cup E_{\text {sync }} \cup E_{\text {conf }}$ where:

- $E_{\text {cont }}=\{(m, n) \mid m, n \in N \wedge \operatorname{Etype}(m, n) \in\{T, F, U\}\}$ is the set of control flow edges.

- $E_{\text {sync }}=\{(m, n) \mid m, n \in N \wedge \operatorname{Etype}(m, n) \in\{\sigma\}\}$ is the set of synchronization edges which show the order enforced by explicit synchronization operations.

- $E_{\text {conf }}=\{(m, n) \mid m, n \in N \wedge \operatorname{Etype}(m, n) \in$ $\left.\left\{\delta^{t}, \delta^{a}, \delta^{0}\right\}\right\}$ is the set of conflict edges. Conflict edges join conflicting basic blocks and are bidirectional.

- Ntype is a function which tells the class of nodes: Ntype : $N \mapsto T_{N}, T_{N}=\{$ Entry, Exit, Pdo, EndPdo, Cobegin, Coend, Condition, Header, Compute, ThreadEntry, ThreadExit\}.

口 Etype is a function which tells the type of edges: Etype : $E \mapsto$ $T_{E}, T_{E}=\left\{T, F, U, \sigma, \delta^{t}, \delta^{a}, \delta^{\circ}\right\}$.

Appendix B

Placing $\pi$-assignments

A CCFG $G=(N, E, N$ type, Etype $)$ is given after renaming.

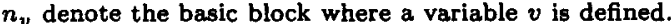

Precede? $(m, n)$ returns true if $m \in \operatorname{Prec}[n]$, otherwise false.

idnum is the next identifying number for a new temporary variable.

Counter $\leftarrow$ idnum

for each node $n \in N$

if $n$ contains a use $v_{0}$ of a shared variable then

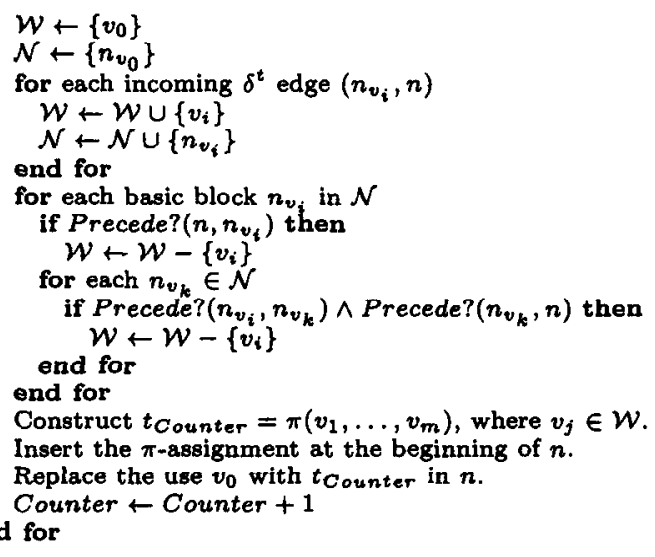

\section{Appendix C}

\section{Proof of Theorem 5.4}

Assume $x$ and $y$ are the first two active and congruent variables but have different values. We will derive a contradiction for each possible operation labeling of the nodes $r$ and $s$ of $x$ and $y$ in the value graph. Without loss of generality, assume also the defining assignment of $x$ has just executed.

Case 1. r's operation label is a constant. $x$ and $y$ should both be constants and equal. A contradiction.

Case 2. r's operation label is op. Because $r$ and $s$ are congruent, $s$ 's operation label must be op. Let $x_{1}$ op $x_{2}$ and $y_{1}$ op $y_{2}$ be the defining expressions of $x$ and $y$. Since $x$ and $y$ have different values, at least one of the pairs $x_{1}$ and $y_{1}$ or $x_{2}$ and $y_{2}$ has different values. Without loss of generality, let $x_{1}$ and $y_{1}$ be such a pair. Since $x$ and $y$ are congruent, $x_{1}$ and $y_{1}$ must be congruent. Moreover, $x_{1}$ and $y_{1}$ must be active variables and have different values. This contradicts to the assumption that $x$ and $y$ are the first two active and congruent variables but have different values.

Case 3. r's operation label is $\phi_{n}$ and $n$ is not a loop header. Because they are congruent, $s^{\prime} s$ operation label must also be $\phi_{n}$. Thus, the defining assignments for $x$ and $y$ occur at the same node. Let $\phi_{n}\left(x_{1}, \ldots, x_{m}\right)$ and $\phi_{n}\left(y_{1}, \ldots, y_{m}\right)$ be the defining expressions in the assignments to $x$ and $y$. Then, $x_{i}$ and $y_{i}$ for some $i$ give different values to $x$ and $y$. In addition, $x_{i}$ and $y_{i}$ must be active variables. This contradicts the assumption.

Case 4. r's operation label is $\phi_{n}$ and $n$ is a loop header. Because $r$ and $s$ are congruent, $s$ 's operation label must also be $\phi_{n}$. Thus, the defining assignments for $x$ and $y$ occur at the same node. Moreover, the innermost loop containing the defining assignment of $x$ and the innermost loop containing the defining assignment of $y$ are the same, i.e., $x$ and $y$ are defined in the same loop. If $x$ and $y$ have different values before the first iteration of the loop, one pair of the congruent variables corresponding to the children of $r$ and $s$, which are related to non-back edge have different values before the loop. If $x$ and $y$ have different values after the $m_{t h}$ iteration of the loop, then one pair of the congruent variables corresponding to the children of $r$ and $s$, which are related to the back edge of the loop have different values before the end of $m_{t h}$ iteration. Either case contradicts the assumption.

Case 5. r's operation label is $\pi$. Let $\pi\left(x_{1}, \ldots, x_{m}\right)$ be the defining expression in the assignment for the node $r$. Then, $x_{i}$ for come $i$ gives the value to $x$. By the definition of congruence, $x_{i}$ is congruent to both $x$ and $y$. In addition, $x_{i}$ is already active. This contradicts the assumption.

Case 6. r's operation label is $\psi$. Same as Case 5.

We did not consider I/O operations, but the proof can be easily extended to $\mathrm{I} / \mathrm{O}$ operations. 